\title{
MCM7 serves as a prognostic marker in diffuse-type gastric adenocarcinoma and siRNA-mediated knockdown suppresses its oncogenic function
}

\author{
WEI KANG ${ }^{1-4}$, JOANNA H.M. TONG ${ }^{1-3}$, ANTHONY W.H. CHAN ${ }^{1}$, \\ ALFRED S.L. CHENG ${ }^{5}$, JUN YU ${ }^{2}$ and KAFAI TO ${ }^{1-4}$
}

\begin{abstract}
${ }^{1}$ Department of Anatomical and Cellular Pathology, State Key Laboratory in Oncology in South China, Prince of Wales Hospital, ${ }^{2}$ Institute of Digestive Disease, Partner State Key Laboratory of Digestive Disease, ${ }^{3}$ Li Ka Shing Institute of Health Science, Sir Y.K. Pao Cancer Center, ${ }^{4}$ Shenzhen Research Institute, The Chinese University of Hong Kong, Shenzhen, ${ }^{5}$ School of Biomedical Sciences, The Chinese University of Hong Kong, Hong Kong, SAR, P.R. China
\end{abstract}

Received December 10, 2013; Accepted February 5, 2014

DOI: $10.3892 /$ or.2014.3094

\begin{abstract}
MCM7 (mini-chromosome maintenance protein 7 ) is essential for the initiation of genomic replication as a component of the pre-replication complex. The present study aimed to analyze its expression, clinical significance and biological functions in gastric adenocarcinoma (GAC). The MCM7 protein was upregulated in all 9 GAC cell lines. In 6 paired primary GACs, MCM7 was upregulated in tumor compared with the corresponding non-tumorous gastric tissues. In normal gastric epithelium tissue, MCM7 was strictly expressed in the proliferative compartment. MCM7 knockdown by siRNA in gastric cancer cell line AGS and NCI-N87 significantly suppressed cell proliferation, inhibited monolayer colony formation, reduced cell invasion and induced late apoptosis. Its nuclear expression correlated with advanced age and poorer disease specific survival in diffuse-type GACs. All the findings supported that MCM7 might play an oncogenic role in gastric tumorigenesis. It serves as a potential prognostic marker and therapeutic target in diffuse-type GACs.
\end{abstract}

\section{Introduction}

Gastric cancer consists of primary malignant lesions in any part of the stomach and causes $\sim 870,000$ deaths worldwide per year (1). The highest incidences are in eastern Asia (China, Japan, Korea), South America and Eastern Europe (2). Most stomach cancer is caused by H.pylori infection (3). The mech-

Correspondence to: Dr Kafai To, Department of Anatomical and Cellular Pathology, State Key Laboratory in Oncology in South China, Prince of Wales Hospital, The Chinese University of Hong Kong, 30-32 Ngan Shing Street, Shatin, Hong Kong, SAR, P.R. China E-mail:kfto@cuhk.edu.hk

Key words: mini-chromosome maintenance protein 7, gastric adenocarcinoma, prognostic marker, siRNA anism by which $H$. pylori induces stomach cancer potentially involves chronic inflammation or the action virulence factors such as CagA (4). Dietary factors including some foods with high sodium such as smoked foods, salted fish and pickled vegetables are associated with higher risk (5). Smoking increases the risk of developing gastric cancer significantly (6). Other factors associated with increased risk are autoimmune atrophic gastritis, intestinal metaplasia and genetic factors (7). Gastric cancers are overwhelmingly adenocarcinoma (8) and gastric adenocarcinoma (GAC) is a malignant epithelial tumor which originates from glandular epithelium of the gastric mucosa. According to Lauren classification, it is divided as two major types, intestinal-type and diffuse-type.

MCM7 is one of the highly conserved mini-chromosome maintenance proteins (9) that are essential for the initiation of genomic replication. The hexametric protein complex formed by the MCM proteins is a key component of the pre-replication complex (pre-RC) and involved in the formation of replication forks and in the recruitment of other DNA replication related proteins $(10,11)$. Some studies have suggested that MCM4, 6 and 7 complexes contain DNA helicase activity $(12,13)$ and act as a DNA unwinding enzyme. MCM7 amplification and overexpression in several human malignancies has been identified (14-16), and its function in cancer development is gradually revealed.

However, in GAC, MCM7 has not been functionally investigated and evaluated on its clinical significance. In the present study, we provide the first evidence that MCM7 overexpression is correlated with diffuse-type GAC patient survival and siRNA mediated downregulation of MCM7 in gastric cancer cell lines has anti-oncogenic effect.

\section{Materials and methods}

Cell lines and primary GAC samples. The 9 GAC cell lines (MKN28, KATOIII, MKN45, SNU16, SNU1, MKN7, MKN1, NCI-N87 and AGS) (17), were cultured in RPMI-1640 (Gibco) supplemented with $10 \%$ fetal bovine serum (FBS; EU Gibco), $100 \mathrm{U} / \mathrm{ml}$ penicillin and $10 \mu \mathrm{g} / \mathrm{ml}$ streptomycin in a humidified 
atmosphere of $5 \% \mathrm{CO}_{2}$ at $37^{\circ} \mathrm{C}$. Archival tissue blocks from 123 patients with diffuse-type GAC were retrieved from the Department of Anatomical and Cellular Pathology, Prince of Wales Hospital (PWH), Hong Kong. Frozen tissues from another 6 pairs of primary GACs and corresponding nontumorous gastric mucosa were collected from patients who underwent gastectomy before any therapeutic intervention. The AJCC (7th edition) was used for cancer staging.

RNA extraction and $q R T-P C R$. Total RNA was extracted using RNeasy Mini kit according to the manufacturer's instruction (Qiagen). High-Capacity cDNA reverse transcription kits (Applied Biosystems) were applied to achieve cDNAs. For qRT-PCR, SYBR-Green PCR Master Mix (Applied Biosystems) was applied for MCM7 expression (sense, ACTT GTGGAATGGCAGGAAG and antisense, CTAGGCATCC CACTCAAAGG). The normal gastric mRNA was from Ambion (AM 7996). The relative expression level was normalized by glyceraldehyde-3-phosphate dehydrogenase (GAPDH) and calculated using the $2^{-\Delta \Delta C t}$ method. Quantitative PCR was performed in triplicate reaction wells including positive and negative controls.

Immunohistochemical staining and scoring. Immunohistochemical staining of MCM7 was performed in $4 \mu \mathrm{m}$-thick sections. After de-waxing in xylene, sections were rehydrated, rinsed in distilled water, and incubated in $3 \% \mathrm{H}_{2} \mathrm{O}_{2}$ solution for 25 min to block endogenous peroxidase activities. Antigen retrieval was done by using pressure cooker with $10 \mathrm{nM}$ citrate buffer ( $\mathrm{pH} \mathrm{6.0)}$ ) for a total of $30 \mathrm{~min}$ (high power $10 \mathrm{~min}$ followed by low power $20 \mathrm{~min}$ ). The primary antibody MCM7 (1:1,000, CDC47 Ab-2; NeoMarkers, Fremont, CA, USA) was applied at $4^{\circ} \mathrm{C}$ overnight and chromogen development was performed using the standard avidin-biotin method (Dako). The nuclear expression of MCM7 was scored by estimating proportion of tumor cells with positive nuclear staining into 4 different groups (negative, none; weak, $\leq 10 \%$; moderate, 10 to $\leq 25 \%$; strong, $>25 \%$ ).

Western blot analysis. Protein was extracted from the GAC cell lines, normal gastric epithelium tissues together with 6 paired primary samples using RIPA lysis buffer with proteinase inhibitor. Protein mixed $(20 \mu \mathrm{g})$ with 5X SDS loading buffer was loaded per lane, separated by $12 \%$ SDS-polyacrylamide gel electrophoresis. MCM7 protein was detected with MCM7 antibody (1:50, CDC47 Ab-2; NeoMarkers). Cleaved-PARP (Asp214) (1:1,000, \#9541; Cell Signaling Technology, Danvers, MA, USA) was also evaluated to check late apoptosis.

In vitro functional study assays. Transfection was performed using Lipofectamine 2000 transfection reagent (Invitrogen) and siMCM7 (SI05064276 and SI05064283; Qiagen) at the concentration of $25 \mathrm{nM}$. Scramble siRNA was included as control. Cell proliferation was assessed using CellTiter 96 ${ }^{\circledR}$ Non-Radioactive Cell Proliferation Assay (MTT) (Promega, Madison, WI, USA) according to the manufacturer's instructions. The absorbance at $570 \mathrm{~nm}$ was measured and documented for the 5-day MTT proliferation analysis (Victor3; Perken-Elmer, Waltham, MA, USA). Monolayer colony formation assay was performed as described in our previous report (18). We used 6-well plates for this assay, 3 for siMCM7 and 3 for siScramble. The time-point was 8 days after transfection. Colonies were fixed with $70 \%$ ethanol for 15 min and stained with $2 \%$ crystal violet. Colonies with cell number of $>50$ cells/colony were counted. Cell invasion assay was performed in a 24 -well invasion chamber (BD BioCoat Matrigel Invasion Chamber; BD Biosciences). Cells were harvested from the culture dishes $24 \mathrm{~h}$ after transfection, washed three times with culture medium containing $1 \%$ FBS, and resuspended in medium with $1 \%$ FBS. Then, $300 \mu \mathrm{l}$ of the cell suspension $\left(1 \times 10^{5}\right.$ cells $)$ was added into the Transwells, with $500 \mu \mathrm{l}$ of culture medium containing $10 \%$ FBS in the lower chamber. After $24-\mathrm{h}$ incubation at $37^{\circ} \mathrm{C}$, cells that invaded through the Matrigel membrane were fixed with $100 \%$ methanol for 2 min and stained with $1 \%$ Toluidine blue for another $2 \mathrm{~min}$. Cells on the underside of the membrane were counted from 3 microscope fields and the average number recorded.

Statistical analysis. In the functional assays like MTT proliferation, monolayer colony formation and cell invasion, Student's t-test was used to compare the phenotype difference of siMCM7 knockdown cells and siScramble transfected cells. Correlations between MCM7 expression with other clinicopathologic parameters were evaluated by non-parametric Spearman's rho rank test. The Kaplan-Meier method was used to estimate the survival rates according to MCM7 nuclear staining. For the parameters statistically significant in the univariate survival analysis $(\mathrm{P}<0.05)$, the multivariate survival was achieved by the Cox regression analysis. All statistical analysis was performed by SPSS software (version 16.0; SPSS Inc.). A two-tailed P-value of $<0.05$ was considered to indicate a statistically significant result.

\section{Results}

Upregulation of MCM7 in gastric cell lines and primary gastric tumors. The MCM7 mRNA expression levels were higher in 6 GAC cell lines (KatoIII, MKN45, SNU16, MKN1, NCI-N87 and AGS) than it in the normal gastric tissue as shown in Fig. 1A. The upregulation of MCM7 in the GAC cells was further confirmed by western blot analysis. High MCM7 protein expression was observed in all GAC cell lines compared with the 3 normal gastric mucosal samples from patients who underwent weight reduction gastrectomy (Fig. 1B). Comparing tumor with the paired non-tumorous mucosa, upregulation of MCM7 protein was observed in all 6 tumor samples by western blot analysis (Fig. 1C). In normal gastric epithelium tissues, only cells from neck region (proliferation compartment) showed MCM7 nuclear expression by immunohistochemistry (Fig. 1D). Immunohistochemical analysis of 5 paired primary GAC samples showed positive nuclear staining of MCM7 in the tumor tissues but not in the non-tumorous gastric glandular epithelium (Fig. 1E).

MCM7 knockdown in GAC cells exerts anti-oncogenic effect in vitro. Upregulation of MCM7 in GACs suggested a potential tumorigenic role of MCM7 in cancer development. To further elucidate the functional role it plays, we investigated the effect of MCM7 knockdown by siRNA in vitro. 
A

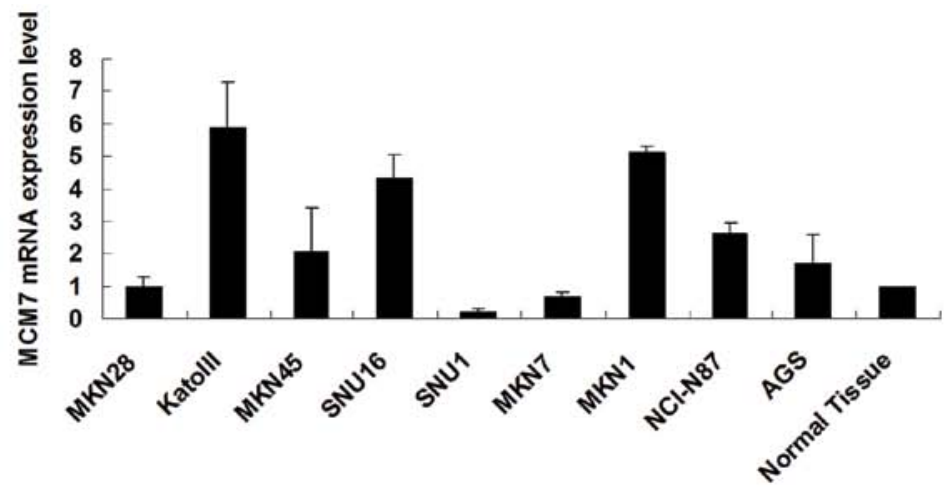

B

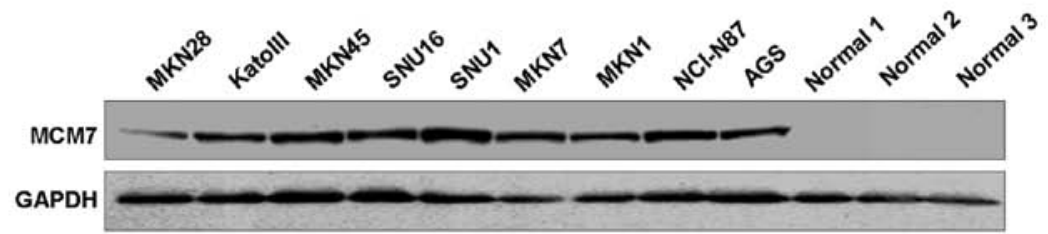

C

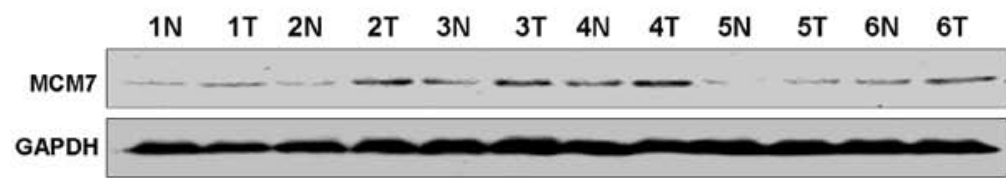

D
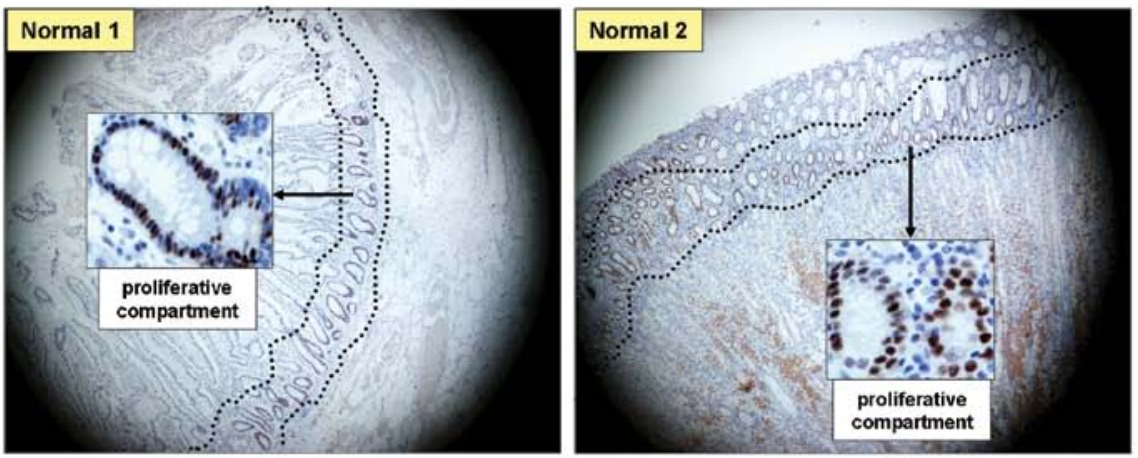

E
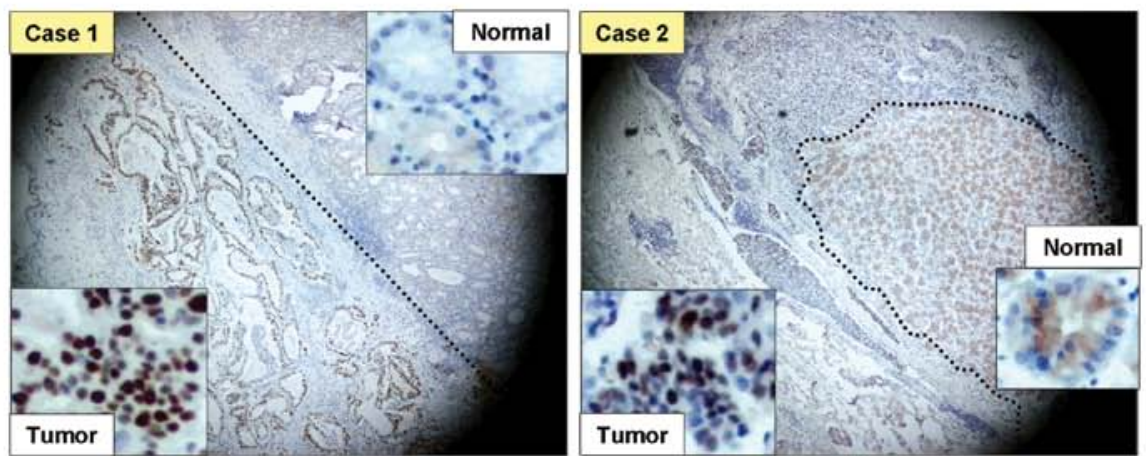

Figure 1. MCM7 upregulation in gastric cancer cell lines and primary GACs. (A) MCM7 mRNA expression in 9 gastric cancer cell lines compared with normal gastric mRNA. The experiment was done in triplicates. (B) MCM7 protein expression in gastric cancer cell lines and 3 normal gastric mucosal tissues from patients with weight reduction gastrectomy. (C) Western blot analysis of MCM7 in 6 pairs of gastric tumors (T) and the corresponding non-tumorous mucosa (N). (D) Immunohistochemical staining of MCM7 in normal gastric epithelium. Only cells in proliferative compartment show MCM7 nuclear staining (original magnification, x400). (E) Immunohistochemical staining of MCM7 in paired GAC samples (normal, adjacent normal tissue; tumor, tumor tissue; original magnification, $\mathrm{x} 400)$.

MCM7 were knocked down by 2 specific siMCM7s in AGS and NCI-N87 with high transfection efficiency (Fig. 2A). The 5-day MTT assay indicated that the MCM7 knock- down reduced cell proliferation rates to significant levels (AGS, 32.1 and 30.0\%; NCI-N87, 40.5 and 43.6\%; $\mathrm{P}<0.001$ ) (Fig. 2B) compared with siScramble control groups. The 
A

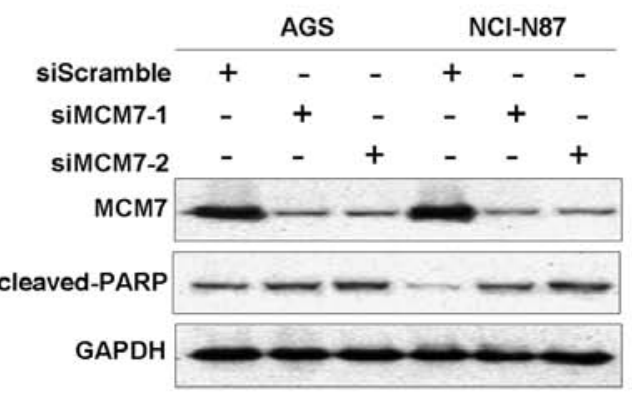

B

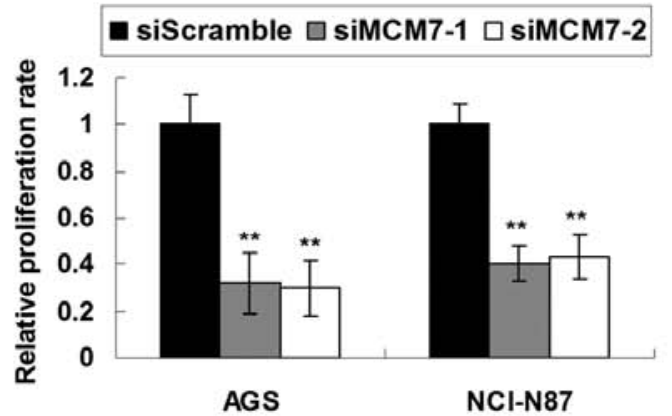

C

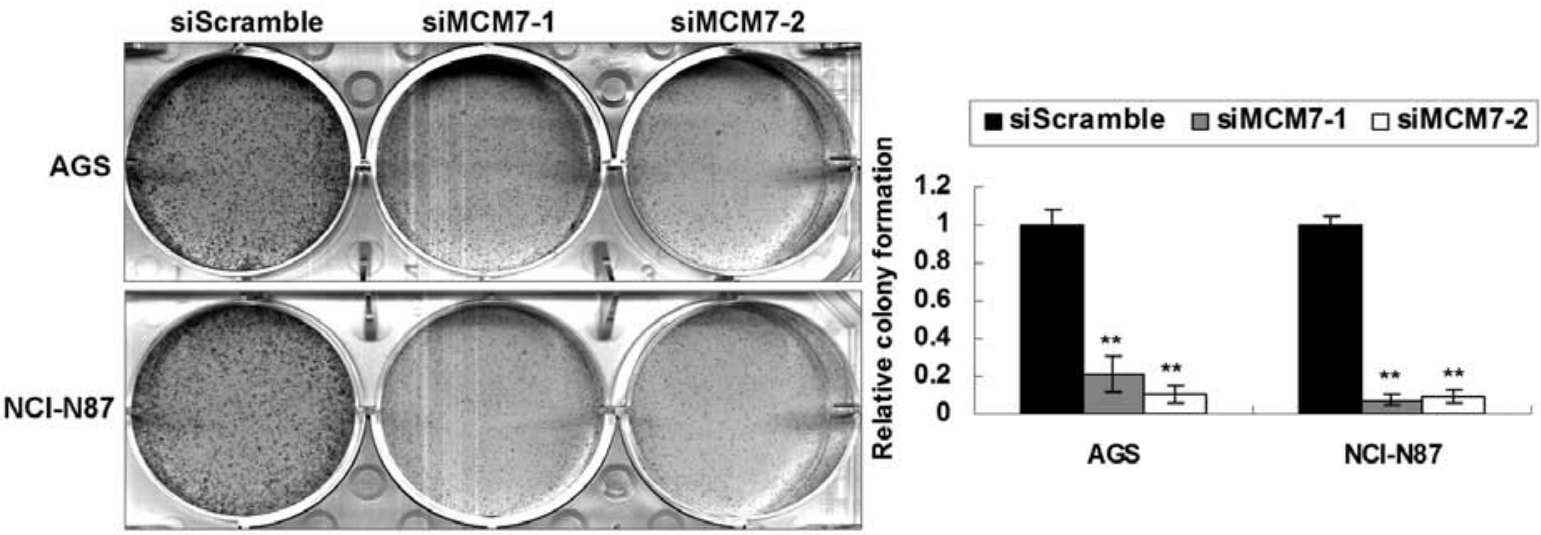

D
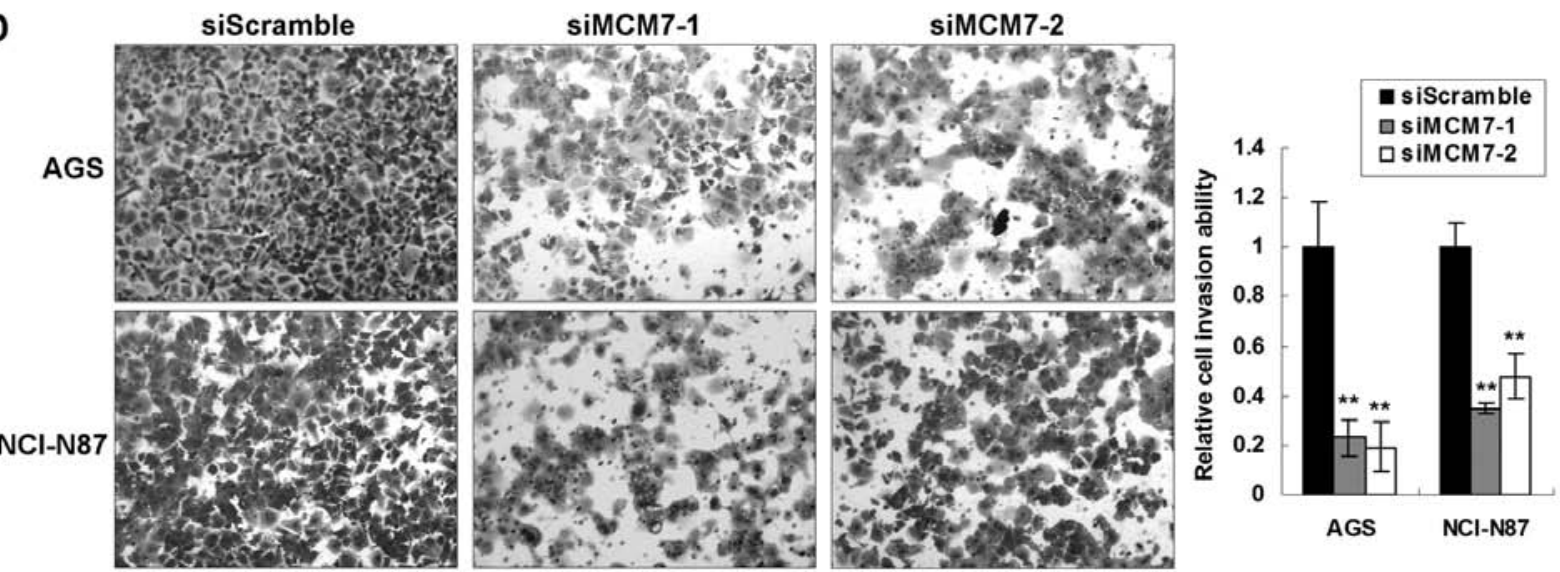

Figure 2. MCM7 knockdown by siRNA in GAC cell lines. (A) Transfection with siMCM7 reduced MCM7 protein expression and activated cleaved-PARP in AGS and NCI-N87 cells. (B) Five-day MTT assays revealed MCM7 siRNA suppressed gastric cancer cell proliferation (**P<0.001). (C) Monolayer colony formation assays suggested transfection with siMCM7 reduced monolayer colony formation in AGS and NCI-N87 cells $\left({ }^{* *} \mathrm{P}<0.001\right)$. The experiments were done in triplicates. (D) Representative photos of invaded cells are shown. The number of invaded cells was lower in MCM7 siRNA group than it in control group in AGS and NCI-N87 cells $\left({ }^{* *} \mathrm{P}<0.001\right)$. The cell number was counted in three view fields randomly and the error bars represent standard deviations.

viability change induced by siMCM7 was also confirmed by anchorage-dependent monolayer colony formation assays. A significant reduction of colony number and colony size was observed in cells transfected with siMCM7 compared with the siScramble group (reduced to 21.3 and $10.6 \%$ in AGS; 7.6 and $9.2 \%$ in NCI-N87; P<0.001) (Fig. 2C). In cell motility assays using BD BioCoat chamber, we confirmed that the invaded cell number was significantly lower in the siMCM7 transfected cells than the control groups (impaired to 23.0 and $19.1 \%$ in AGS; 34.9 and $47.6 \%$ in NCI-N87; P<0.001) (Fig. 2D), suggesting that MCM7 was involved in the invasion of cancer cells. Moreover, siMCM7 induced late apoptosis in AGS and NCI-N87 cells which was represented by activation of cleaved-PARP (Fig. 2A).
MCM7 overexpression correlates with poor survival in diffuse-type primary GACs. In total 267 GAC samples, MCM7 expression had no correlation with survival $(\mathrm{P}=0.724)$, thus, we checked its prognostic correlation according to histological type (intestinal and diffuse) and stage (early and advanced) separately. We found only in the diffuse-type GAC, MCM7 correlates with poor survival.

MCM7 was predominantly expressed in the nuclei of diffuse-type tumor cells (Fig. 3A). Of the 123 diffuse-type GAC samples, 16 cases (13.0\%) had MCM7 negative nuclear staining, 11 cases $(8.9 \%)$ had weak staining, 44 cases (35.8\%) had moderate staining and 52 cases $(42.3 \%)$ had strong staining. No significant survival difference was observed between the 4-grade MCM7 expression by univariate analysis $(\mathrm{P}=0.071$; 
A

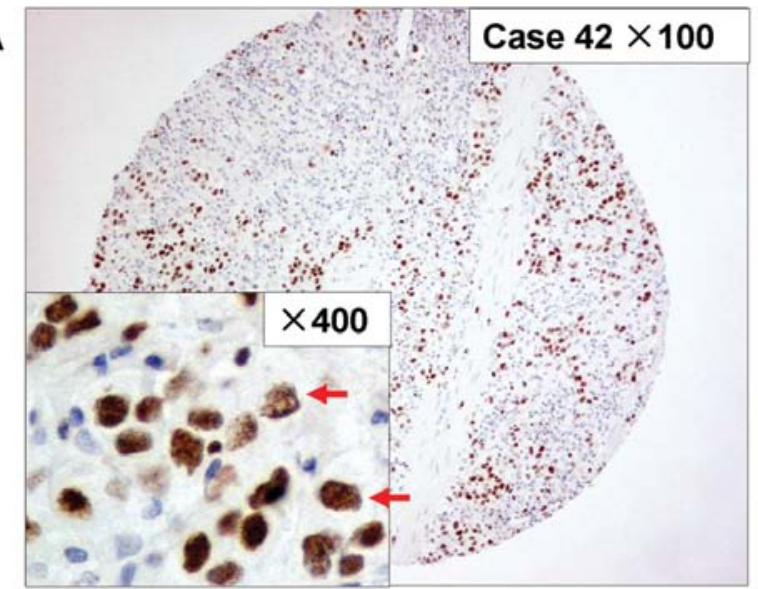

B

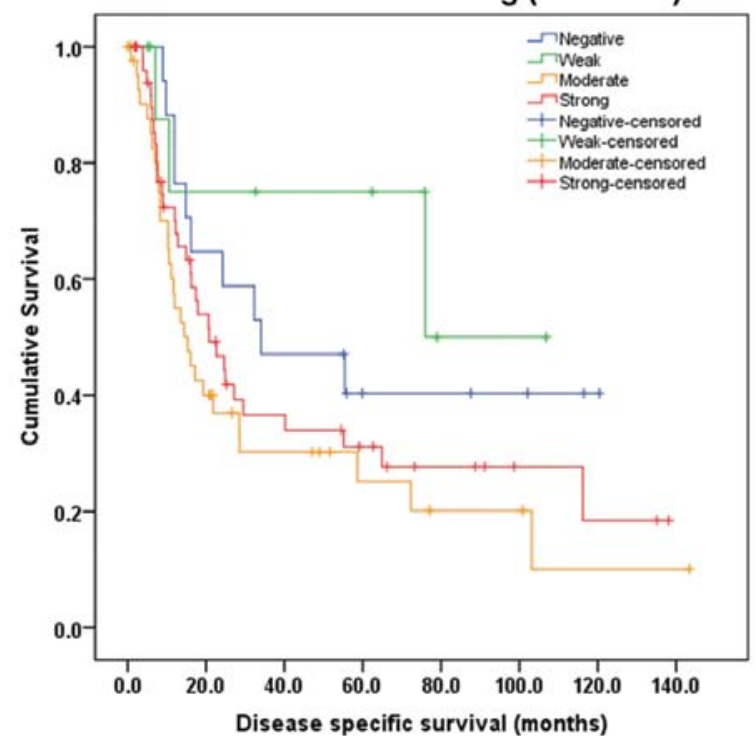

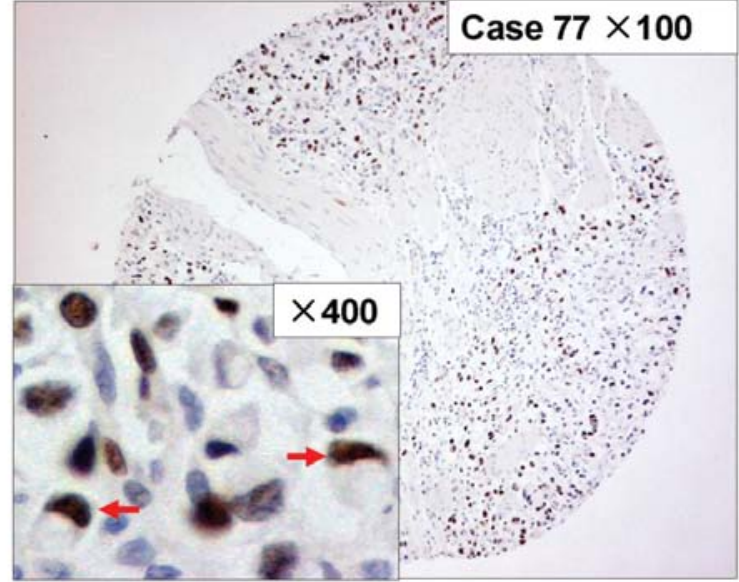

C
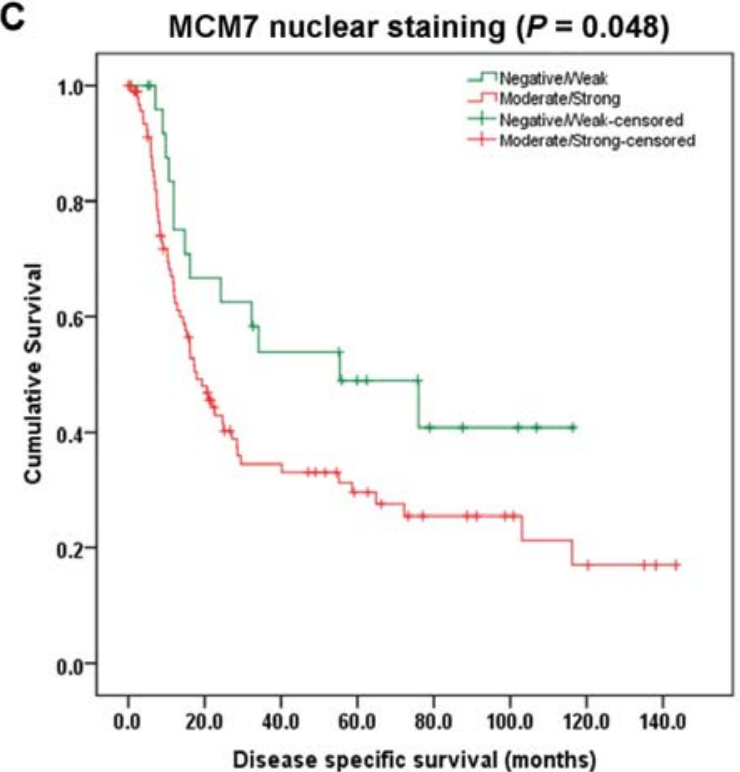

Figure 3. MCM7 nuclear expression correlates with poor survival in diffuse-type gastric tumors. (A) Representative figures of MCM7 immunohistochemical staining in primary diffuse-type GACs, case 42 and case 77. MCM7 nuclear expression in tumor cells and signet ring cells is indicated by a red arrowhead (original magnification, x100; insertion, x400). (B) Kaplan-Meier survival of diffuse-type GACs on MCM7 nuclear staining (negative, 16 cases; weak, 11 cases; moderate, 44 cases; strong, 52 cases; $\mathrm{P}=0.071$ ). (C) Kaplan-Meier survival curve of diffuse-type GACs according to MCM7 nuclear staining status (negative and weak expression groups, 27 case; moderate and strong expression group, 96 cases; $\mathrm{P}=0.048$ ).

Fig. 3B). Then, we stratified negative and weak staining cases as negative/weak MCM7 expression group (27/123, 22.0\%), and moderate and strong cases as moderate/strong MCM7 expression group (96/123, 78.0\%). MCM7 nuclear expression correlates with poor survival based on these groupings $(\mathrm{P}=0.048$; Fig. 3C).

We further investigated the clinicopathological correlation of MCM7 in diffuse-type GACs. Table I summarizes the correlation of MCM7 with clinicopathological parameters in diffuse-type GAC patients. MCM7 expression only correlated with advanced age ( $>60$ years; $\mathrm{P}=0.015)$. Univariate analysis (Table II) indicated that MCM7 expression in diffuse-type GACs associated with poor disease-specific survival $(\mathrm{P}=0.048)$. Advanced age and stage were (T, N and $\mathrm{M}$, respectively) also correlated with poor survival $(\mathrm{P}<0.05)$. By multivariate Cox proportional hazards regression analysis (Table II), only stage ( $\mathrm{T}, \mathrm{N}$ and $\mathrm{M}$, respectively) was independently associated with disease-specific survival $(\mathrm{P}<0.05)$.

\section{Discussion}

In gastric cancer, MCM7 was first screened from mRNA expression microarray (Fig. 4A) and array-CGH (comparative genomic hybridization, Fig. 4B) in 9 GAC cell lines. The MCM7 mRNA expression showed positive correlation with DNA copy number change (Fig. 4C), indicating MCM7 DNA copy number gain was partly responsible for its mRNA upregulation in GAC, and van Dekken et al (19) screened MCM7 using the same method in gastroesophageal junction adenocarcinomas due to the DNA amplification and mRNA upregulation. MCM7 gene on chromosome 7q22 contains an intronic miRNA miR-106b-25 cluster in intron 13 (20). MCM7 oncogenicity may be linked, at least in part, to overexpression of the hosted miRNAs which targets TGF- $\beta$ tumor suppressor pathway in gastric cancer (21).

MCM7 has been reported to be upregulated in many types of cancer and functions as oncogene in muscle- 
Table I. Correlation of MCM7 nuclear expression with clinicopathological features.

\begin{tabular}{|c|c|c|c|}
\hline & \multicolumn{3}{|c|}{ Diffuse-type GAC cases $(n=123)$} \\
\hline & $\begin{array}{c}\text { Negative/weak } \\
\text { No. }(\%)\end{array}$ & $\begin{array}{c}\text { Moderate/strong } \\
\text { No. }(\%)\end{array}$ & P-value \\
\hline \multicolumn{4}{|l|}{ Gender } \\
\hline Male & $16(20.5)$ & $62(79.5)$ & 0.655 \\
\hline Female & $11(24.4)$ & $34(75.6)$ & \\
\hline \multicolumn{4}{|c|}{ Age (years) } \\
\hline$\leq 60$ & $18(32.7)$ & $37(67.3)$ & 0.015 \\
\hline$>60$ & $9(13.2)$ & $59(86.8)$ & \\
\hline \multicolumn{4}{|l|}{ Grade } \\
\hline 1 & 0 & 0 & 0.575 \\
\hline 2 & $0(0.0)$ & $4(100.0)$ & \\
\hline 3 & $27(22.7)$ & $92(77.3)$ & \\
\hline \multicolumn{4}{|l|}{ Stage } \\
\hline 1 & $3(18.8)$ & $13(81.3)$ & 0.879 \\
\hline 2 & $2(33.3)$ & $4(66.7)$ & \\
\hline 3 & $9(23.7)$ & $29(76.3)$ & \\
\hline 4 & $13(20.6)$ & $50(79.4)$ & \\
\hline \multicolumn{4}{|l|}{ Stage $(\mathrm{T})$} \\
\hline 1 & $1(10.0)$ & $9(90.0)$ & 0.770 \\
\hline 2 & $6(20.7)$ & $23(79.3)$ & \\
\hline 3 & $18(23.4)$ & $59(76.6)$ & \\
\hline 4 & $2(28.6)$ & $5(71.4)$ & \\
\hline \multicolumn{4}{|l|}{ Stage (N) } \\
\hline 0 & $6(33.3)$ & $12(66.7)$ & 0.521 \\
\hline 1 & $3(13.6)$ & $19(86.4)$ & \\
\hline 2 & $9(22.0)$ & $32(78.0)$ & \\
\hline 3 & $9(21.4)$ & 33 (78.6) & \\
\hline \multicolumn{4}{|l|}{ Stage (M) } \\
\hline 0 & $20(21.3)$ & 74 (78.7) & 0.799 \\
\hline 1 & $7(24.1)$ & $22(75.9)$ & \\
\hline \multicolumn{4}{|c|}{ Lymph node } \\
\hline 0 & $6(33.3)$ & $12(66.7)$ & 0.224 \\
\hline 1 & $21(20.0)$ & $84(80.0)$ & \\
\hline \multicolumn{4}{|l|}{ H.pylori } \\
\hline Absence & $14(20.9)$ & $53(79.1)$ & 0.503 \\
\hline Presence & 13 (27.7) & $34(72.3)$ & \\
\hline
\end{tabular}

Significant P-value in bold and italic format.

invasive bladder cancer (22), non-small cell lung cancer $(23,24)$, Hodgkin lymphoma (25), prostate cancer $(15)$, liver cancer (26), meningiomas (27), colorectal cancer $(28,29)$, medulloblastoma (30) and oral squamous cell carcinoma (31). By siRNA-mediated knockdown of MCM7 in GAC cell lines, the cell proliferation and invasion were inhibited, thus MCM7 knockdown exerts anti-oncogenic effect in vitro. This result was concordant with functional studies in other types of cancer. In medulloblastoma, exog-

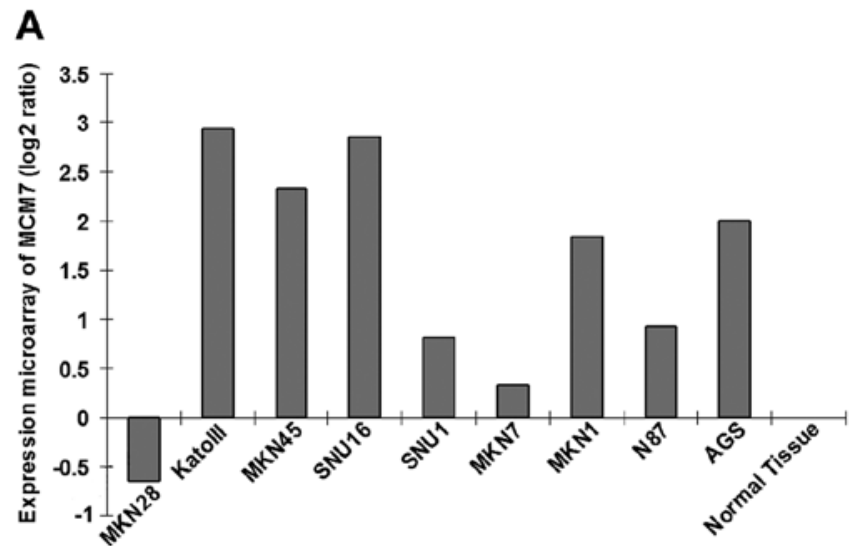

B
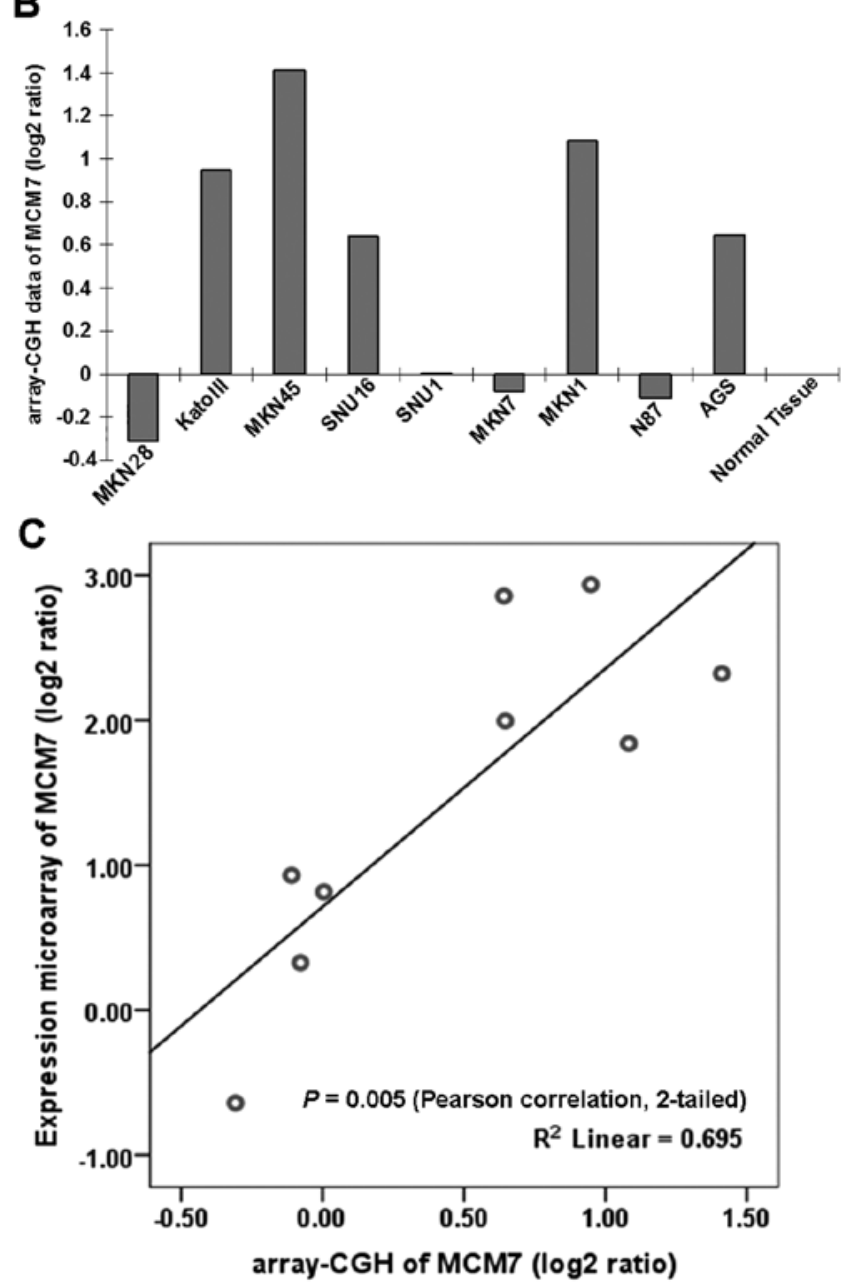

Figure 4. Correlation of expression microarray and array-CGH data of MCM7 in 9 GAC cell lines. (A) Expression microarray data of MCM7 in 9 gastric cancer cell lines normalized with normal gastric epithelium RNA ( $\log 2$ ratio). (B) Array-CGH data of MCM7 normalized with normal gastric epithelium DNA ( $\log 2$ ratio). (C) Correlation of expression microarray and array-CGH in 9 gastric cancer cell lines $(\mathrm{P}=0.005)$.

enous overexpression of MCM7 increased tumor growth and invasiveness, whereas MCM7 knockdown by RNA interference inhibited cell growth and reduced cell migration and invasion (30). MCM7 elimination in prostate cancer and non-small cell lung cancer cell lines also suppressed cell growth. In a mouse model of PC 3 and DU145 xenografts of 
Table II. Univariate and multivariate Cox regression analysis of clinicopathological factors in patients with GAC.

Diffuse-type GAC cases $(n=123)$

Univariate (P-value) Multivariate (P-value)

\begin{tabular}{lrr}
\hline Gender & 0.838 & \\
Age & $\mathbf{0 . 0 0 8}$ & \\
Grade & 0.259 & \\
Stage (T) & $\mathbf{0 . 0 0 2}$ & $\mathbf{0 . 0 2 0}$ \\
Stage (N) & $<\mathbf{0 . 0 0 1}$ & $\mathbf{0 . 0 0 1}$ \\
Stage (M) & $<\mathbf{0 . 0 0 1}$ & $\mathbf{0 . 0 0 7}$ \\
H.pylori & 0.763 & \\
MCM7 & $\mathbf{0 . 0 4 8}$ & \\
\hline
\end{tabular}

Significant $\mathrm{P}$-value in bold and italic format.

prostate cancer, knockdown of MCM7 dramatically reduced the tumor volumes, decreased tumor metastasis and animal mortality (32).

Some reports indicated that MCM7 was a more reliable and useful biomarker in assessing tumor proliferation, aggression and in the prognosis of patients even than the existing markers such as Ki-67 $(33,34)$. In primary diffuse-type GACs, we identified that MCM7 was moderately/strongly stained in 96 of 123 GAC (78.0\%) and its overexpression was closely correlated with advanced age ( $>60$ years). However, MCM7 expression was not detected associated with other clinicopathological factors including clinical grade, stage and lymph node metastasis. In endometrial carcinoma (35) and Hodgkin lymphoma (25), MCM7 overexpression also correlates with advanced age. More importantly, its upregulation correlates with poor survival and predicts poor prognosis, indicating MCM7 might have potential as a biomarker and therapeutic target in diffuse-type GAC.

In conclusion, the present findings indicate that MCM7 expression may be a useful prognosis indicator in patients with diffuse-type GACs and its knockdown by RNA interference quenches its oncogenic property in gastric cancer.

\section{Acknowledgements}

The present study is supported by a National Natural Science Grant of China (81201591).

\section{References}

1. Kelley JR and Duggan JM: Gastric cancer epidemiology and risk factors. J Clin Epidemiol 56: 1-9, 2003.

2. Smith MG, Hold GL, Tahara E and El-Omar EM: Cellular and molecular aspects of gastric cancer. World J Gastroenterol 12: 2979-2990, 2006.

3. Shiota S, Suzuki R and Yamaoka Y: The significance of virulence factors in Helicobacter pylori. J Dig Dis 14: 341-349, 2013.

4. Sepulveda AR: Helicobacter, inflammation, and gastric cancer. Curr Pathobiol Rep 1: 9-18, 2013.

5. Zhong C, Li KN, Bi JW and Wang BC: Sodium intake, salt taste and gastric cancer risk according to Helicobacter pylori infection, smoking, histological type and tumor site in China. Asian Pac J Cancer Prev 13: 2481-2484, 2012.
6. Zhang MW, Jin MJ, Yu YX, et al: Associations of lifestyle-related factors, hsa-miR-149 and hsa-miR-605 gene polymorphisms with gastrointestinal cancer risk. Mol Carcinog 51 (Suppl 1): E21-E31, 2012.

7. Gomceli I, Demiriz B and Tez M: Gastric carcinogenesis. World J Gastroenterol 18: 5164-5170, 2012.

8. Green D, Ponce de Leon S, Leon-Rodriguez E and SosaSanchez R: Adenocarcinoma of the stomach: univariate and multivariate analysis of factors associated with survival. Am J Clin Oncol 25: 84-89, 2002.

9. Kebebew E, Peng M, Reiff E, Duh QY, Clark OH and McMillan A: Diagnostic and prognostic value of cell-cycle regulatory genes in malignant thyroid neoplasms. World J Surg 30: 767-774, 2006.

10. Luo JH: Oncogenic activity of MCM7 transforming cluster. World J Clin Oncol 2: 120-124, 2011.

11. Wei Q, Li J, Liu T, Tong X and Ye X: Phosphorylation of minichromosome maintenance protein 7 (MCM7) by cyclin/ cyclin-dependent kinase affects its function in cell cycle regulation. J Biol Chem 288: 19715-19725, 2013.

12. Ishimi Y: A DNA helicase activity is associated with an MCM4, -6, and -7 protein complex. J Biol Chem 272: 24508-24513, 1997.

13. You Z, Komamura Y and Ishimi Y: Biochemical analysis of the intrinsic Mcm4-Mcm6-mem7 DNA helicase activity. Mol Cell Biol 19: 8003-8015, 1999.

14. Honeycutt KA, Chen Z, Koster MI, et al: Deregulated minichromosomal maintenance protein MCM7 contributes to oncogene driven tumorigenesis. Oncogene 25: 4027-4032, 2006.

15. Ren B, Yu G, Tseng GC, et al: MCM7 amplification and overexpression are associated with prostate cancer progression. Oncogene 25: 1090-1098, 2006.

16. Kan T, Sato F, Ito $\mathrm{T}$, et al: The $m i R-106 b-25$ polycistron, activated by genomic amplification, functions as an oncogene by suppressing p21 and Bim. Gastroenterology 136: 1689-1700, 2009.

17. Kang W, Tong JH, Chan AW, et al: Stathmin1 plays oncogenic role and is a target of microRNA-223 in gastric cancer. PLoS One 7: e33919, 2012

18. Kang W, Tong JH, Chan AW, et al: Yes-associated protein 1 exhibits oncogenic property in gastric cancer and its nuclear accumulation associates with poor prognosis. Clin Cancer Res 17: 2130-2139, 2011.

19. van Dekken H, Tilanus HW, Hop WC, et al: Array comparative genomic hybridization, expression array, and protein analysis of critical regions on chromosome arms $1 \mathrm{q}, 7 \mathrm{q}$, and $8 \mathrm{p}$ in adenocarcinomas of the gastroesophageal junction. Cancer Genet Cytogenet 189: 37-42, 2009.

20. Ambs S, Prueitt RL, Yi M, et al: Genomic profiling of microRNA and messenger RNA reveals deregulated microRNA expression in prostate cancer. Cancer Res 68: 6162-6170, 2008.

21. Petrocca F, Visone R, Onelli MR, et al: E2F1-regulated microRNAs impair TGF $\beta$-dependent cell-cycle arrest and apoptosis in gastric cancer. Cancer Cell 13: 272-286, 2008.

22. Fristrup N, Birkenkamp-Demtroder K, Reinert $\mathrm{T}$, et al: Multicenter validation of cyclin D1, MCM7, TRIM29, and UBE2C as prognostic protein markers in non-muscle-invasive bladder cancer. Am J Pathol 182: 339-349, 2013.

23. Liu YZ, Jiang YY, Hao JJ, et al: Prognostic significance of MCM7 expression in the bronchial brushings of patients with non-small cell lung cancer (NSCLC). Lung Cancer 77: 176-182, 2012.

24. Toyokawa G, Masuda K, Daigo Y, et al: Minichromosome Maintenance Protein 7 is a potential therapeutic target in human cancer and a novel prognostic marker of non-small cell lung cancer. Mol Cancer 10: 65, 2011.

25. Marnerides A, Vassilakopoulos TP, Boltetsou E, et al: Immunohistochemical expression and prognostic significance of CCND3, MCM2 and MCM7 in Hodgkin lymhoma. Anticancer Res 31: 3585-3594, 2011.

26. Zhou YM, Zhang XF, Cao L, et al: MCM7 expression predicts post-operative prognosis for hepatocellular carcinoma. Liver Int 32: 1505-1509, 2012.

27. Saydam O, Senol O, Schaaij-Visser TB, et al: Comparative protein profiling reveals minichromosome maintenance (MCM) proteins as novel potential tumor markers for meningiomas. J Proteome Res 9: 485-494, 2010.

28. Pillaire MJ, Selves J, Gordien K, et al: A 'DNA replication' signature of progression and negative outcome in colorectal cancer. Oncogene 29: 876-887, 2010. 
29. Nishihara K, Shomori K, Fujioka S, et al: Minichromosome maintenance protein 7 in colorectal cancer: implication of prognostic significance. Int J Oncol 33: 245-251, 2008.

30. Lau KM, Chan QK, Pang JC, et al: Minichromosome maintenance proteins 2, 3 and 7 in medulloblastoma: overexpression and involvement in regulation of cell migration and invasion. Oncogene 29: 5475-5489, 2010.

31. Feng CJ, Li HJ, Li JN, Lu YJ and Liao GQ: Expression of Mcm7 and Cdc6 in oral squamous cell carcinoma and precancerous lesions. Anticancer Res 28: 3763-3769, 2008.

32. Shi YK, Yu YP, Tseng GC and Luo JH: Inhibition of prostate cancer growth and metastasis using small interference RNA specific for minichromosome complex maintenance component 7. Cancer Gene Ther 17: 694-699, 2010.
33. Fujioka S, Shomori K, Nishihara K, et al: Expression of minichromosome maintenance 7 (MCM7) in small lung adenocarcinomas (pT1): Prognostic implication. Lung Cancer 65: 223-229, 2009.

34. Ishino $\mathrm{H}$, Hara $\mathrm{Y}$, Takekoshi $\mathrm{S}$, et al: $\mathrm{Ki}-67$ and minichromosome maintenance-7 (MCM7) expression in canine pituitary corticotroph adenomas. Domest Anim Endocrinol 41: 207-213, 2011.

35. Li SS, Xue WC, Khoo US, et al: Replicative MCM7 protein as a proliferation marker in endometrial carcinoma: a tissue microarray and clinicopathological analysis. Histopathology 46: 307-313, 2005. 